\title{
Avaliação Eletromiográfica de Músculos da Cintura Escapular e Braço Durante a Realização de Exercícios Com Extremidade Fixa e Carga Axial
}

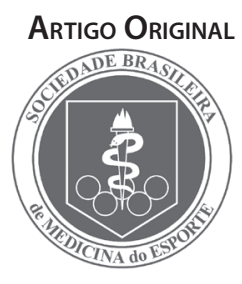

\author{
Electromyographic Assessment of Scapular Girdle and Arm Muscles \\ During Exercises With Fixed Boundary and Axial Load
}

Daniel Pereira Cardoso de Brum Marcel Morais de Carvalho'

Helga Tatiana Tucci ${ }^{2}$

Anamaria Siriani de Oliveira

1. Centro Universitário do Triângulo, Uberlândia, Brasil

2. Universidade de São Paulo, FMRP/ USP, Ribeirão Preto, São Paulo, Brasil.

\section{Endereço para correspondência: Profa. Dra. Anamaria Siriani de \\ Oliveira \\ Fisioterapia - Av. Bandeirantes, 3900 - CEP 14049-900 - Ribeirão Preto \\ - São Paulo \\ Fone: (016) 3602-4415 \\ E-mail: siriani@fmrp.usp.br}

Submetido em 25/05/2007

Versão final recebida em 24/04/2008 Aceito em 28/04/2008

\begin{abstract}
RESUMO
O objetivo deste estudo foi comparar a atividade eletromiográfica de músculos da cintura escapular e braço entre os exercícios wall-press $90^{\circ}$, wall-press $45^{\circ}$, bench-press e push-up, realizados com a extremidade distal do segmento fixa sobre uma superfície estável e em esforço isométrico máximo. Participaram desta pesquisa 20 voluntários do sexo masculino, sedentários e sem história de trauma ou doenças na extremidade superior. A atividade elétrica da cabeça longa do músculo bíceps braquial, da porção anterior do músculo deltóide, da porção clavicular do músculo peitoral maior e do músculo serrátil anterior foi registrada por eletromiografia de superfície. O teste modelo estatístico ANOVA e o teste post-hoc de Tukey foram utilizados para determinar diferenças entre os valores de RMS de cada músculo e em cada exercício, normalizados pela contração isométrica voluntária máxima. Os resultados do presente estudo demonstram que o músculo bíceps braquial apresentou a menor atividade eletromiográfica em comparação com os demais músculos avaliados em todos os exercícios, enquanto a porção anterior do músculo deltóide e o serrátil anterior mostraram maior atividade eletromiográfica em relação aos demais músculos nos exercícios bench-press e push-up. Esses achados demonstram que não houve semelhança na atividade eletromiográfica dos músculos avaliados durante os exercícios, porém, houve coativação muscular, visto que os exercícios ativaram todos os músculos, mesmo que em diferentes níveis. Os exercícios estudados não são indicados para ativar o músculo bíceps braquial, mas os exercícios bench-press e push-up o são para ativar a porção anterior do músculo deltóide e o serrátil anterior; o wall-press $90^{\circ}$ e o wall-press $45^{\circ}$ são indicados para ativar a porção anterior do músculo trapézio.
\end{abstract}

Palavras-chave: eletromiografia, cadeia cinética fechada, ombro.

\section{ABSTRACT}

The objective of this study was to compare the electromyography activity of scapular and arm muscles in the wall-press $90^{\circ}$, wall-press $45^{\circ}$, bench-press and push-up exercises, accomplished with the distal extremity of the segment on a stable surface and in maximum isometric effort. Twenty male sedentary (23 \pm 7 years), and without trauma history or diseases in the upper extremity volunteers participated in this research. The electric activity of the long head of the muscle biceps brachii, the anterior portion of the deltoid muscle, the clavicular portion of the pectoralis major and the serratus anterior muscle was registered by electromyography surface. The ANOVA and Tukey post hoc were used to determine differences between the RMS values of each muscle and in each exercise, normalized by the maximal voluntary isometric contractions. The results of the present study demonstrated that the biceps brachii muscle presented the smallest electromyographic activity in comparison to the other muscles in all exercises, while the anterior portion of the deltoid and the serratus anterior muscles presented larger electromyography activity in relation to the other muscles in the bench-press and push-up exercises. These findings demonstrate that there was not similarity in the electromyography activity of the evaluated muscles during the exercises; however, there was a muscular coactivation, since the exercises activated all muscles, even if at different levels. The studied exercises are not recommended for activation of the biceps brachii muscle; however, the benchpress and push-up exercises are recommended to activate the anterior portion of deltoid and serratus anterior muscles. Moreover, the wall-press $90^{\circ}$ and the wall-press $45^{\circ}$ are recommended for activation of the upper trapezius muscle.

Keywords: electromyography, closed kinetic chain, shoulder. 


\section{INTRODUÇÃO}

Exercícios em cadeia cinética fechada têm sido sugeridos na prática clínica por serem considerados mais funcionais e seguros e por promoverem estímulos proprioceptivos e menor força de cisalhamento em relação aos exercícios feitos em cadeia cinética aberta ${ }^{(1,2)}$. Entretanto, a classificação dos exercícios cinesioterapêuticos em cadeia cinética aberta e cadeia cinética fechada tem recebido críticas quanto à especificidade desses conceitos, principalmente quando aplicados aos exercícios voltados à extremidade superior ${ }^{(3)}$.

A classificação dos exercícios em "cadeia cinética fechada" (CCF) ou "cadeia cinética aberta" (CCA) foi proposta por Steindler ${ }^{(4)}$, que usou esses termos para classificar os exercícios de acordo com a condição da extremidade distal do segmento, ou seja, se fixa ou móvel. Assim sendo, o termo CCA descreve exercícios realizados com a extremidade distal do segmento livre, enquanto o termo CCF descreve exercícios nos quais a extremidade distal do segmento é fixa e possui resistência externa que impede ou restringe o movimento, condição na qual o movimento em uma articulação produz movimento nas demais ${ }^{(4)}$. Entretanto, definir os exercícios em CCA ou CCF pode ser difícil(4), pois muitos deles podem ser uma combinação dessas duas classificações $^{(5)}$

Dessa forma, Dilman et al. ${ }^{(6)}$ propuseram uma nova classificação para exercícios baseada na condição da extremidade, se fixa ou móvel, e na presença ou ausência da carga no segmento distal da extremidade. Os autores levantaram a hipótese de que exercícios com biomecânica similar realizados com a mesma quantidade de carga seriam capazes de produzir atividade eletromiográfica semelhante em músculos motores primários. Sendo assim, sugeriam que os exercícios deveriam ser definidos com base na sua análise biomecânica e na quantificação da carga imposta ao segmento, em vez de ser classificados em CCA e CCF.

Considerando a necessidade da estabilização do complexo articular do ombro e os conceitos existentes sobre cadeia cinética, Lephart e Henry ${ }^{(7)}$ propuseram uma nova classificação, denominada Sistema de Classificação Funcional, que compreende exercícios destinados à extremidade superior, classificados de acordo com características funcionais e ações neuromusculares comuns. Esse sistema incorporou outras considerações, além da condição da extremidade e da presença ou ausência de carga, sendo elas a direção da carga (axial ou rotacional), a magnitude da resistência (alta velocidade e baixa resistência ou baixa velocidade e alta resistência), a ação muscular (coativação, aceleração ou desaceleração) e a função neuromuscular (ativação voluntária ou reação muscular). Assim, esse sistema classificou os exercícios em: 1) extremidade fixa - carga axial (EFCA); 2) extremidade móvel - carga axial (EMCA); 3) extremidade móvel - carga rotacional (EMCR); e 4) extremidade móvel sem carga (EMSC).

Segundo Lephart e Henry ${ }^{(7)}$, cada condição proposta nesse sistema inclui aspectos específicos do sistema neuromuscular necessários para restaurar a estabilidade funcional do complexo articular do ombro, tornando mais específica a elaboração de um protocolo de reabilitação. Outra consideração feita pelos autores é que o conhecimento das condições neuromusculares e biomecânicas relacionadas a cada condição faz com que o clínico possa elaborar atividades adequadas a cada estágio de reabilitação.

De acordo o Sistema de Classificação Funcional, exercícios em EFCA são realizados com alta resistência e baixa velocidade, produzem reação neuromuscular ativa ou reativa e ação muscular de coativação ou desaceleração, sendo capazes de gerar estabilização dinâmica, compressão articular, propriocepção e mínimas forças de cisalhamento, vantagens associadas à direção axial da carga ou da sustentação de peso corpo- ral(6-8). Dessa forma, a análise desse tipo de exercício possui relevância clínica em decorrência das ações musculares e neurofisiológicas que são capazes de produzir.

Estudos têm procurado classificar os exercícios de acordo com a atividade elétrica da musculatura envolvida ${ }^{(6,9-11)}$, justificada pela necessidade do conhecimento do quanto um determinado exercício consegue ativar os músculos do complexo articular do ombro, visto que os estabilizadores dinâmicos são os principais responsáveis pela estabilidade articular e pela biomecânica normal dessa região(12-14). Além disso, a análise de quanto um exercício ativa um músculo ou grupo muscular pode tornar mais clara sua inclusão em um programa de reabilitação ${ }^{(15,16)}$. Assim sendo, a eletromiografia de superfície tem sido uma das ferramentas utilizadas em estudos recentes sobre protocolos cinesioterapêuticos voltados à extremidade superior ${ }^{(11,17-}$ ${ }^{18)}$, tanto em indivíduos saudáveis ${ }^{(15,16)}$ quanto em indivíduos com alguma disfunção, como síndrome do impacto e instabilidade glenoumeral ${ }^{(17)}$

Assim sendo, o objetivo deste estudo foi avaliar e comparar, em voluntários saudáveis, a atividade eletromiográfica de músculos da cintura escapular e braço nos exercícios wall-press $90^{\circ}$, wall-press $45^{\circ}$, bench-press e push-up, classificados com EFCA. A hipótese deste trabalho é de que esses exercícios produzem níveis de atividade eletromiográfica semelhantes por ser realizados com biomecânica similar, ou seja, com a extremidade distal fixa em base estável e com o mesmo valor de carga axial.

\section{MÉTODOS}

\section{Descrição da amostra}

Foram avaliados 20 voluntários do sexo masculino ( $23 \pm 7$ anos), sedentários e destros que, de acordo com os critérios de inclusão, não apresentaram história de trauma na cintura escapular e nos membros superiores, hipotrofia visível e pontos- gatilhos nos músculos avaliados. Também foram realizados testes específicos para o manguito rotador (Neer, Jobe e Hawkins), para instabilidade do tendão da porção longa do bíceps braquial (Yegarson), instabilidade ântero-posterior (Rockwood) e multidirecional (Sulco) e teste para avaliar a integridade do músculo subescapular (Gerber), com o objetivo de excluir os voluntários com sinais clínicos de síndrome do impacto e instabilidade articular do ombro. Os voluntários que apresentaram restrição de movimento na articulação do ombro e/ou presença de arco doloroso durante os movimentos de elevação do braço foram excluídos da pesquisa pelo fato desses fatores provocarem variações nos padrões eletromiográficos nos diferentes músculos da cintura escapular e do braço ${ }^{(19)}$. Foram considerados sedentários os voluntários que realizaram até duas atividades físicas diferentes, repetidas no máximo três vezes por semana, não específicas para membros superiores e sem caráter de treinamento físico desportivo. Os voluntários assinaram o termo de consentimento livre e esclarecido, segundo a Resolução n 196/96 do Conselho Nacional da Saúde, aprovado pelo Comitê de Ética do Hospital das Clínicas da Faculdade de Medicina de Ribeirão Preto da Universidade de São Paulo - USP.

\section{Exercícios propostos}

Os exercícios wall-press $90^{\circ}$, wall-press $45^{\circ}$, bench-press e push-up, classificados em EFCA, foram realizados de forma isométrica sobre uma superfície estável (figura 1, A-D). 0 exercício wall-press $90^{\circ}$ foi realizado com o voluntário em posição ortostática, o membro superior dominante em $90^{\circ}$ de flexão de ombro no plano sagital e punho em $90^{\circ}$ de extensão. $O$ wall-press $45^{\circ}$ foi realizado com o mesmo posicionamento do wall-press $90^{\circ}$, alterando somente a angulação de flexão do 

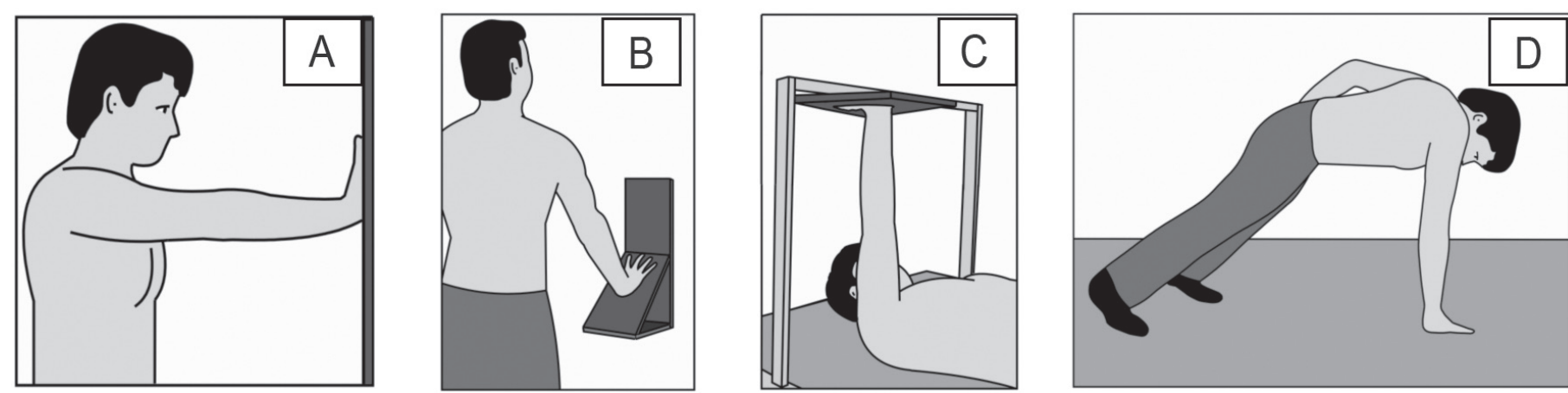

Figura 1. Exercícios avaliados feitos com a extremidade distal fixa e carga axial (EFCA). A - Wall-press 90 ; B - Wall-press $45^{\circ}, \mathbf{C}-$ Bench-press; D - Push-up.

ombro para $45^{\circ}$. Para a realização do exercício bench-press, o voluntário foi posicionado em decúbito dorsal, com os pés apoiados na maca, membro superior dominante em $90^{\circ}$ de flexão de ombro e $90^{\circ}$ de extensão de punho. Por fim, o exercício push-up foi realizado com o voluntário em decúbito ventral, apoiado sobre os pés, posição neutra de tronco e com o membro superior dominante em $90^{\circ}$ de flexão de ombro e punho em $90^{\circ}$ de extensão.

\section{Instrumentação}

Os registros eletromiográficos foram captados por eletrodos de superfície ativos simples diferencial (EMG System do Brasil, São José dos Campos, SP, Brasil), com ganho de 20 vezes, compostos por duas barras retangulares e paralelas de prata pura (Ag) $(10 \times 1 \times 1 \mathrm{~mm})$, impedância de entrada de $10 \mathrm{G} \Omega$ e razão de rejeição em modo comum $($ RRMC) $>130 \mathrm{~dB}$. Os sinais eletromiográficos de superfície foram coletados por cinco canais do eletromiógrafo Myosystem Br-1 (Datahominis Tecnologia Ltda., Uberlândia, MG, Brasil. O eletromiógrafo possui aterramento e aquisição simultânea comum para todos os canais, filtro digital com passa-faixa de 20 a $500 \mathrm{~Hz}$, impedância de $10 \mathrm{G} \Omega$ em modo diferencial, 12 bits de faixa de resolução dinâmica, faixa de amplitude de -10V a +10V, placa conversora analógico-digital (A/D) e freqüência de amostragem por canal de $4 \mathrm{KHz}$. O processamento dos sinais eletromiográficos foi realizado pelas rotinas do programa Myosistem Br-1 versão 2.9. (Datahominis Tecnologia Ltda., Uberlândia, MG, Brasil).

\section{Procedimento experimental}

Após a avaliação e inclusão do voluntário na pesquisa, foram registrados os sinais eletromiográficos da cabeça longa do músculo bíceps braquial, da porção anterior do músculo deltóide, da porção clavicular do músculo peitoral maior e do músculo serrátil anterior. O posicionamento dos eletrodos foi orientado pela European Recommendations for Surface Electromyography do Projeto SENIAN(20). Um eletrodo de referência retangular $(33 \times 31 \mathrm{~mm})$ posicionado sobre a região do manúbrio esternal foi utilizado com a função de diminuir o efeito de interferências eletromagnéticas e outros ruídos de aquisição do sinal eletromiográfico. Antes da colocação dos eletrodos, foram realizadas tricotomia, abrasão e posterior assepsia da pele com algodão embebido em álcool, com o objetivo de diminuir a impedância da pele e alcançar a máxima fixação do eletrodo. O eletrodo de referência foi acoplado com gel condutor e tanto os eletrodos de registro quanto o de referência foram fixados por fita adesiva. Três contrações isométricas voluntárias máximas foram realizadas para cada músculo em posicionamento de prova de função muscular (21). Cada CIVM teve duração de quatro segundos e com intervalos entre si de dois minutos. O valor máximo das três CIVMs serviu como valor de referência para normalização dos valores de root mean square (RMS) para cada um dos músculos estudados. Os exercícios foram realizados uma hora após a coleta das CIVMs para evitar possível fadiga muscular, que poderia influenciar a captação do sinal eletromiográfico durante os exercícios. Nesse período, o voluntário permaneceu no laboratório, sentado em uma cadeira e os eletrodos não foram removidos para evitar que sua recolocação pudesse ser um fator de alteração na coleta eletromiográfica. A seqüência de realização dos exercícios foi aleatória e cada um deles foi repetido três vezes, com duração de quatro segundos cada repetição. Um período de dois minutos foi dado entre as contrações e um de cinco minutos entre cada exercício para prevenir o efeito da fadiga no sinal eletromiográfico. Os exercícios foram realizados com esforço isométrico máximo, ou seja, foi solicitado que o voluntário realizasse a maior força isométrica possível e a mantivesse durante o tempo de coleta. O examinador esteve atento para evitar que os voluntários realizassem qualquer movimento compensatório e a instrução dos exercícios e o comando verbal na realização das CIVM foram padronizados e dados sempre pelo mesmo examinador.

\section{Análise dos dados}

O sinal eletromiográfico não foi janelado, ou seja, os valores da amplitude eletromiográfica obtidos durante os quatro segundos de coleta de cada um dos exercícios são representados em valores de root means square (RMS), sugerido entre as possíveis formas de apresentação de tal variável pelas normas de padronização para estudos eletromiográficos de superfície ${ }^{(20)}$. O valor médio de RMS de cada músculo estudado foi normalizado pelo valor máximo de RMS de três registros da CIVM do mesmo músculo, ou seja, pela razão entre o valor médio nos exercícios estudados pelo valor máximo obtido dos registros da CIVM. Dessa forma, os resultados do trabalho serão apresentados em unidade arbitrária (UA). Essa terminologia UA é decorrente do procedimento de normalização, ou seja, da divisão de um sinal eletromiográfico bruto por outro sinal eletromiográfico bruto escolhido como fator de normalização. Essa divisão elimina a unidade microvolts $(\mu \mathrm{V})$, deixando os valores em unidade arbitrária (UA). Os valores de RMS dos quatro exercícios avaliados foram comparados entre si através da análise de variância (ANOVA) e do pós-teste de Tukey, com nível de significância de $5 \%$ ou $p<0,05$.

\section{RESULTADOS}

Os valores de RMS normalizados de cada músculo estudado nos exercícios wall-press $90^{\circ}$, wall-press $45^{\circ}$, bench-press e push-up estão representados nas figuras de 2 a 5 . 
No exercício wall-press $90^{\circ}$, o bíceps braquial apresentou diferença estatisticamente significativa, com menor amplitude eletromiográfica, quando comparado com a porção anterior do músculo deltóide, a porção superior do músculo trapézio e o serrátil anterior $(p<0,05)$, mas sua amplitude não foi estatisticamente significativa quando comparado com o peitoral maior ( $p<0,05$ ) (figura 2). Quando comparados os valores eletromiográficos normalizados no exercício wall-press $45^{\circ}$, o teste estatístico demonstrou que o músculo bíceps braquial apresentou diferença estatisticamente significativa, com menor amplitude eletromiográfica quando comparado com a porção anterior do músculo deltóide, as fibras superiores do músculo trapézio, serrátil anterior e porção clavicular do peitoral maior $(p<0,05)$ (figura 3).

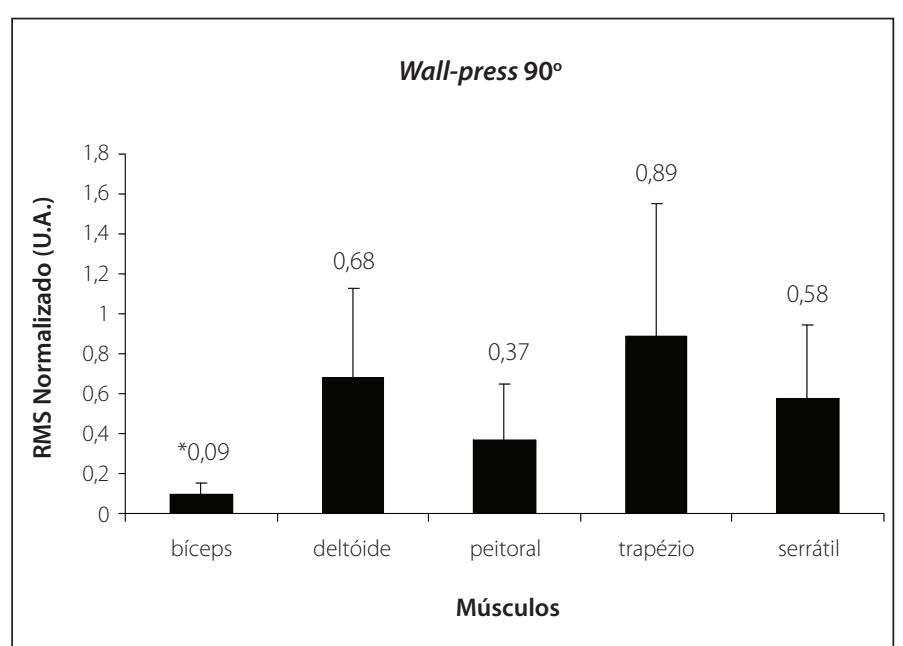

Figura 2. Valores médios e desvios-padrão dos valores de RMS normalizados pela contração isométrica voluntária máxima dos músculos bíceps braquial, das fibras anteriores do músculo deltóide, da porção clavicular do peitoral maior, das fibras superiores do músculo trapézio e serrátil anterior registrados durante a realização do exercício wall-press $90^{\circ}(n=20)$.

*Valores significativos $(p<0.05)$

*Diferença significativa em relação a todos os músculos, exceto porção clavicular do peitoral maior

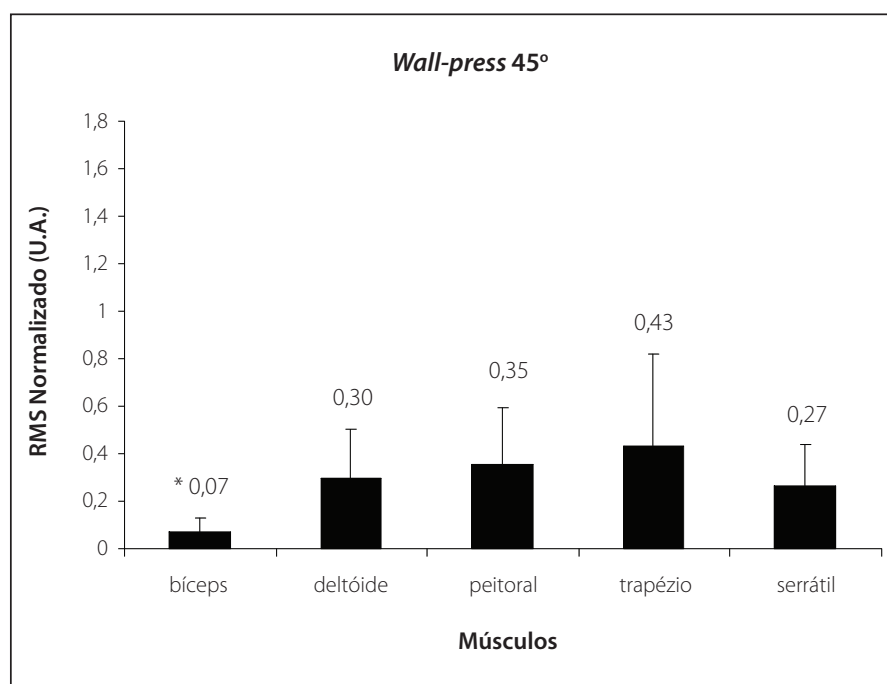

Figura 3. Valores médios e desvios-padrões dos valores de RMS normalizados pela contração isométrica voluntária máxima dos músculos bíceps braquial, das fibras anteriores do músculo deltóide, da porção clavicular do peitoral maior, das fibras superiores do músculo trapézio e serrátil anterior registrados durante a realização do exercício wall-press $45^{\circ}(n=20)$.

*Valores significativos $(p<0.05)$

*Diferença significativa em relação a todos os músculos, exceto porção clavicular do peitoral maior
Na comparação entre os valores de RMS normalizados dos músculos avaliados no exercício push-up e bench-press, não houve diferença estatisticamente significativa entre o músculo serrátil anterior e a porção anterior do músculo deltóide (figuras 4 e 5). Porém, no exercício push-up, houve diferença estatisticamente significativa, com maior amplitude eletromiográfica do serrátil anterior e da porção anterior do deltóide quando comparados com o bíceps braquial e a porção clavicular do peitoral maior ( $p<0,05)$. No bench-press houve diferença estatisticamente significativa, com maior amplitude eletromiográfica do serrátil anterior e da porção anterior do deltóide quando comparados com o bíceps braquial e as fibras superiores do músculo trapézio $(p<0,05)$.

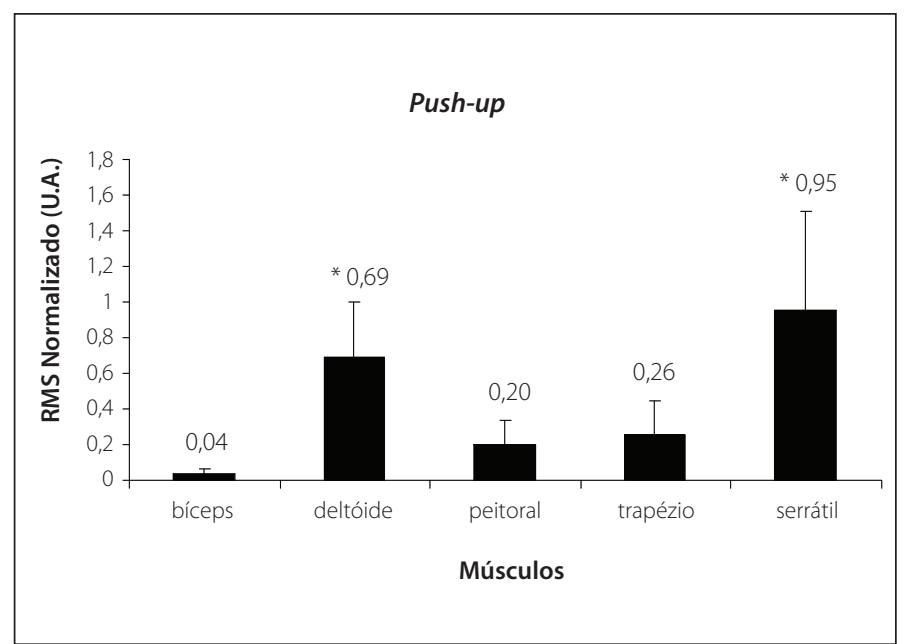

Figura 4. Valores médios e desvios-padrões dos valores de RMS normalizados pela contração voluntária máxima dos músculos bíceps braquial, das fibras anteriores do músculo deltóide, da porção clavicular do peitoral maior, das fibras superiores do músculo trapézio e serrátil anterior registrados durante a realização do exercício push-up $(\mathrm{n}=20)$.

*Valores significativos $(p<0.05)$

*Diferença significativa em relação ao bíceps braquial e a porção clavicular do peitoral maior e a porção superior do trapézio

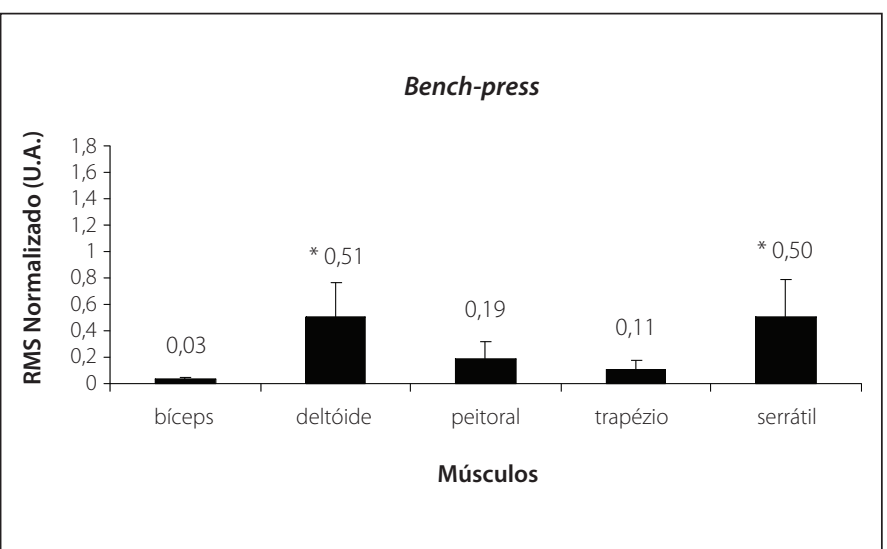

Figura 5. Valores médios e desvios-padrões dos valores de RMS normalizados pela contração voluntária máxima dos músculos bíceps braquial, das fibras anteriores do músculo deltóide, da porção clavicular do peitoral maior, das fibras superiores do músculo trapézio e serrátil anterior registrados durante a realização do exercício bench-press $(n=20)$.

*Valores significativos $(p<0.05)$

*Diferença significativa em relação ao bíceps braquial e a porção superior do trapézio

**Diferença significativa em relação ao bíceps braquial e a porção superior do trapézio 


\section{DISCUSSÃO}

Os resultados do presente estudo demonstraram que os exercícios avaliados não apresentaram níveis de atividade semelhante em todos os músculos analisados, não confirmando a hipótese inicial deste trabalho. Além disso, as semelhanças encontradas em um exercício não necessariamente foram as mesmas verificadas nos demais.

Apesar de não haver a semelhança eletromiográfica sob hipótese, a análise dos dados demonstrou que durante a realização dos quatro exercícios ocorreu coativação, pois houve atividade eletromiográfica em todos os músculos avaliados, mesmo que tenha sido em maior ou menor amplitude. Esses achados concordam com pesquisas prévias, que também encontraram atividade eletromiográfica similar em parte dos músculos estudados quando padronizaram a condição da extremidade e quantidade de carga empregada ${ }^{(6,11)}$. Entretanto, esses achados discordam das características musculares e neurofisiológicas esperadas para os exercícios em EFCA, pois a coativação da musculatura avaliada deveria promover similaridade eletromiográfica entre os valores de amplitude eletromiográfica, já que os exercícios avaliados possuem características que permitem classificá-los dentro da mesma categoria de exercícios (EFCA). Resultados semelhantes foram encontrados na pesquisa de Oliveira et al.(11). Essa diferença pode estar relacionada ao posicionamento do voluntário durante a realização dos exercícios, que pode ter estimulado de maneira distinta mecanorreceptores presentes nas estruturas capsulares e ligamentares, em conseqüência da posição da extremidade superior em relação à gravidade. Isso pode ser justificado pela semelhança nos achados entre os exercícios wall-press $90^{\circ}$ e wall-press $45^{\circ}$ e entre os exercícios bench-press e push-up.

O músculo bíceps braquial demonstrou menor amplitude eletromiográfica quando comparado com os demais músculos em todos os exercícios estudados, com valores de amplitude eletromiográfica inferiores a 10\% da atividade registrada durante a CIVM. A baixa ativação desse músculo pode estar relacionada com a máxima congruência óssea e estabilidade articular do cotovelo decorrente da posição do membro superior em rotação neutra do braço e extensão completa do cotovelo(22) e da existência de forças compressivas transmitidas do úmero à cavidade glenóidea em exercícios com carga axial, que resultam em estabilidade compressiva na articulação glenoumeral(23,24). Também não existiu nenhum movimento de flexão do antebraço durante a realização dos exercícios, não havendo necessidade nem de estabilização e nem de ação motora primária desse músculo, o que também poderia justificar sua baixa atividade elétrica. De acordo com os resultados encontrados e do ponto de vista clínico, os exercícios avaliados nesta pesquisa não são apropriados para ativar o músculo bíceps braquial.

As fibras superiores do músculo trapézio demonstraram elevada atividade durante os exercícios wall-press $90^{\circ}$ e wall-press $45^{\circ}$, cerca de $90 \%$ da atividade obtida durante a CIVM. Esses achados podem estar relacionados com o posicionamento do voluntário durante o exercício, pois diferentemente do bench-press e do push-up, o wallpress possui um componente de força rotacional que exige maior atividade desse músculo para manter a rotação superior da escápula. Alta atividade eletromiográfica desse músculo também foi encontrada por Oliveira et al. ${ }^{(11)}$ no exercício wall-press, justificada pelos autores como decorrente da exigência de um maior recrutamento de unidades motoras para manter o ritmo escapular e, pela sua posição encurtada nesse exercício, pois um maior número de unidades motoras poderia estar abaixo do sítio de detecção do eletrodo, au- mentando a amplitude do sinal eletromiográfico. Além disso, nossos resultados concordam com Lephart e Henry ${ }^{(7)}$, que consideram importante a análise da direção da carga, se axial ou rotacional, ao classificar exercícios.

Durante o push-up e o bench-press, foi observada maior atividade eletromiográfica das fibras anteriores do músculo deltóide e do serrátil anterior quando comparados com os demais músculos, possivelmente causada pela ação sinérgica dessa musculatura na flexão do ombro(24), um dos movimentos necessários para a manutenção da posição do braço nesses exercícios. Além disso, esses músculos estão em posição encurtada nos exercícios push-up e no bench-press, o que pode ter aumentado o número de unidades motoras ativas sob o eletrodo de captação, elevando a amplitude do sinal eletromiográfico(26).

A elevada atividade eletromiográfica do serrátil anterior no pushup e no bench-press também pode ser justificada pela direção axial da carga, que é transmitida posteriormente à articulação escapulotorácica e contrabalanceada pela ação estabilizadora do serrátil anterior. Dessa forma, maior atividade desse músculo é necessária para garantir a fixação adequada da escápula no tórax ${ }^{(27)}$. Esse resultado demonstra a importância de incluir aspectos não considerados por Lephart e Henry ${ }^{(7)}$, como diferenças na ativação muscular decorrentes da direção da carga e do movimento escapular realizado durante o exercício, que poderia causar ações neuromusculares distintas nos músculos avaliados, dependendo de sua ação, se agonista, sinergista ou antagonista. Outra consideração interessante para a prática clínica é que o push-up com apoio nos joelhos ativa menos o serrátil anterior em comparação com o push-up realizado com apoio dos pés ${ }^{(17,28)}$, demonstrando que a base de apoio do voluntário pode ser referência para ativar em maior ou menor grau o músculo serrátil anterior.

Observando os resultados do presente estudo foi possível verificar que o exercício wall-press $45^{\circ}$ foi o que teve maior similaridade nos níveis de atividade eletromiográfica entre os músculos estudados, aproximadamente 25-30\% da CIVM correspondente. Esse achado pode ser elucidado de acordo com a proposta de Dilman et al. ${ }^{(7)} \mathrm{e}$ Blackard et al. ${ }^{(9)}$, que afirmam que exercícios com similaridades biomecânicas, isto é, mesma quantidade e direção da carga, deveriam exibir comparáveis níveis de atividade muscular. No presente estudo, todos os exercícios foram realizados com carga axial, esforço isométrico máximo e mesmo posicionamento da extremidade superior; entretanto, a descarga de peso pode ter variado, o que justificaria a não semelhança nos diferentes níveis de atividade eletromiográfica entre os exercícios.

Segundo o Sistema de Classificação proposto por Lephart e Henry ${ }^{(7)}$, exercícios feitos com carga axial e extremidade distal fixa são capazes de produzir coativação dos pares de forças escapulares e umerais, promover a estabilização dinâmica do ombro, propriocepção, mínimas forças de cisalhamento e alguma semelhança com atividades funcionais dos membros superiores, fatores que devem ser considerados em fases iniciais da reabilitação de doenças no complexo articular do ombro, principalmente as relacionadas com a glenoumeral, como instabilidades, subluxações e síndrome do impacto. Entretanto, devemos levar em consideração que o exercício push-up deve ser realizado com cautela em indivíduos com instabilidade posterior do complexo articular do ombro, visto que a posição do voluntário durante este exercício é a de maior ocorrência de deslocamento/subluxação da ombro, ou seja, em flexão, rotação interna e adução(16). 
O estudo de músculos do manguito rotador teria importante contribuição clínica na análise feita neste trabalho. Entretanto, esses músculos não são superficiais e, por isso, não podem ser avaliados pela eletromiografia de superfície, limitando considerações sobre a atividade dessa musculatura. Entretanto, exercícios com carga axial são capazes de ativar o infra-espinhoso por produzirem uma força de cisalhamento posterior na glenoumeral, levando a aumento da atividade desse músculo para estabilizar a cabeça umeral e prevenir seu deslocamento posterior ${ }^{(16)}$. Além disso, exercícios que promovam significativa atividade do músculo deltóide anterior são capazes de gerar uma ação sinérgica do infra-espinhoso e redondo menor, músculos que controlam a translação superior da cabeça umeral|(13). Outros músculos sinérgistas, como o as fibras posteriores do músculo deltóide, as fibras inferiores do trapézio e o levantador da escápula também deveriam ter sido avaliados.

Outra limitação deste estudo é o não controle da carga utilizada e a não análise de diferentes valores de carga ou de percentagens de esforço isométrico, fatores que poderiam influenciar a atividade eletromiográfica. Sendo assim, pesquisas eletromiográficas futuras deveriam analisar a atividade eletromiográfica de outros músculos sinergistas do movimento escapular, como os rombóides e o elevador da escápula e avaliar a influência que diferentes níveis de esforço ou de carga teriam sobre a amplitude do sinal eletromiográfico. Esses achados poderiam auxiliar na escolha de exercícios para a reabilitação do membro superior.

\section{REFERÊNCIAS BIBLIOGRÁFICAS}

1. Wilk EK, Arrigo CA, Andrews JR. Closed and open kinetic chain exercise for the upper extremity. J Sport Rehabil 1996;5:88-102.

2. Kibler WB. Shoulder rehabilitation: principles and practice. Med Sci Sports Exerc 1998;30:40-50.

3. KernozekTW, McLean KP, McLean DP. Biomechanical and physiologic factors of kinetic chain exercise in the lower extremity. Orthop Phys Ther Clin N Am 2000;:587-91.

4. Steindler A. Kinesiology of the human body. Springfield, II: Charles C. Thomas, 1973.

5. Panariello RA. The closed kinetic chain in strength training. J Strength Cond Res 1991;13:29-33.

6. Dillman CJ, Murray TA, Hintermeister RA. Biomechanical differences of open and closed chain exercises with respect in the shoulder. J Sport Rehabil 1994;3:228-38.

7. Lephart SM, Henry TJ. The Physiologycal Basis for Open and Closed Hinetic Chain Rehabilitation for the upper extremity. J Sport Rehabil 1996;5:71-87.

8. Borsa PA, Lephard SM, Kocker MS. Functional assessment and rehabilitation of shoulder proprioception for glenoumeral instability. J Sport Rehabil 1994;3:84-104.

9. Blackard DO, Jensen RL, Ebben WP. Use of EMG analysis in challenging kinetic chain terminology. Med Sci Sports Exerc 1999;31(3):443-8.

10. Kuechle DK, Newman SR, Itoi E, Niebur GL, Morrey BF, An KN. The relevance of the moment arm of shoulder muscles with respect to axial rotation of the glenohumeral joint in four positions. Clin Biomech 2000;15:322-9.

11. Oliveira AS, Freitas CMS, Monaretti H, Ferreira F, Noguti R, Bérzin F. Avaliação eletromiográfica de músculos da cintura escapular e braço durante exercícios com carga axial e rotacional. Rev Bras Med Esporte 2006;12:1-5.

12. Pratt NE. Anatomy and Biomechanics of the shoulder.J Hand Ther 1994; 65-76.

13. Sharkey NA, Marder RA. The rotador cuff opposes superior translation of the humeral head. Am J Sports Med 1995;23:301-6.

\section{CONCLUSÃO}

Nessas condições experimentais, podemos observar que não houve atividade eletromiográfica semelhante em todos os músculos estudados e que quando ocorreu similaridade entre alguns músculos, ela não foi mantida em todos os exercícios. Porém, houve coativação entre os músculos estudados porque todos estavam ativos durante os registros eletromiográficos. Em relação á pratica clínica, os exercícios estudados produziram baixa atividade eletromiográfica no bíceps braquial, não sendo os mais indicados para ativar esse músculo. Entretanto, os exercícios push-up e bench-press ativaram significativamente as fibras anteriores do músculo deltóide e o serrátil anterior e os exercícios wall-press $90^{\circ}$ e wall-press $45^{\circ}$ ativaram significativamente as fibras superiores do músculo trapézio e, dessa forma, podem ser utilizados na prática clínica para ativar essa musculatura. Por fim, trabalhos futuros que mensurem a quantidade de carga imposta à extremidade superior, que avaliem a direção dos deslocamentos intra-articulares e a ativação dos músculos do manguito rotador em exercícios para a extremidade superior poderiam auxiliar na escolha de exercícios na reabilitação do complexo articular do ombro.

Todos os autores declararam não haver qualquer potencial conflito de interesses referente a este artigo.
14. Panjabi M. The stabilising system of the spine. Part I. Function, dysfunction, adaption and enchancement. J Spinal Disord 1992;5(4):383-9.

15. Moseley JB, Jobe FW, Pink MM, Perry J, Tibone J. EMG analysis of the scapular muscles during a shoulder rehabilitation program. Am J Sports Med 1992;20:128-34.

16. Uhl TL, Carver TJ, Mattacola CG, Mair SD, Nitz AJ. Shoulder musculature activation during upper extremity weight-bearing exercise. J Orthop Sports Phys Ther 2003;33:109-17.

17. Ludewig PM, Hoff MS, Osowski EE, Meschke SA, Rundquist PJ. Relative Balance of Serratus Anterior and Upper trapezius Muscle Activity During Push-Up Exercises. Am J Sports Med 2004;32:484-93.

18. Gouvali MK, Boudolos K. Dynamic and electromyographical analysis in variants of push-up exercise. J Strength Cond Res 2005;19:146-51.

19. Kelly BT, KirKendall DT, Levy AS, Speer KP. Current research on muscle activity about the shoulder. Instr Course Lect 1997;46:53-66

20. Hermens HJ, Freriks B, Merletti R, Stegeman R, Block J, Rau G, et al. European recommendations for Surface Electromyography - Results of SEIAN Project. Roessing Research and Development, Deliverable 8, 1999

21. Kendal FP, McCreary EK, Provance PG. Músculos - Provas e Funções. 4a ed. São Paulo: Manole, 1995.

22. Lehmkuhl LD, Smith LK. Cinesiologia Clínica de Brunnstrom. $4^{a}$ ed. São Paulo: Manole, 1989.

23. Sarrafian SK. Gross and functional anatomy of the shoulder. Clin Rel Res 1983;1:11-9

24. Sharkey NA, Marder RA. The Rotador Cuff Opposes Superior Translation of the Humeral Head. Am J Sports Med 1995;23:301-6

25. Kapandji Al. Fisiologia Articular - Volume 1. 6a. ed. Rio de Janeiro: Guanabara Koogan, 2007.

26. De Luca CJ. The use of electromyography in biomechanics. J Biomech 1997;13:135-63.

27. Lippert LS. Cinesiologia Clínica para Fisioterapeutas. 3a. ed. Rio de Janeiro: Guanabara Koogan, 2003.

28. Lear $L$, Gross MT. An electromyographical analysisof the scapular stabilizing synergists during a push-up progression. J Orthop Sports Phys Ther 1998;28:146-57. 